\title{
Safety of Ultrasound-Guided Transrectal Extended Prostate Biopsy in Patients Receiving Low-Dose Aspirin
}

\author{
Ioannis Kariotis, Prodromos Philippou, Demetrios Volanis, Efraim Serafetinides, Demetrios \\ Delakas
}

Department of Urology, “Asklipieion” General Hospital, Athens, Greece

\begin{abstract}
Purpose: To determine whether the peri-procedural administration of low-dose aspirin increases the risk of bleeding complications for patients undergoing extended prostate biopsies.

Materials and Methods: From February 2007 to September 2008, 530 men undergoing extended needle biopsies were divided in two groups; those receiving aspirin and those not receiving aspirin. The morbidity of the procedure, with emphasis on hemorrhagic complications, was assessed prospectively using two standardized questionnaires.

Results: There were no significant differences between the two groups regarding the mean number of biopsy cores $(12.9 \pm$ 1.6 vs. $13.1 \pm 1.2$ cores, $\mathrm{p}=0.09)$. No major biopsy-related complications were noted. Statistical analysis did not demonstrate significant differences in the rate of hematuria $(64.5 \%$ vs. $60.6 \%, \mathrm{p}=0.46)$, rectal bleeding $(33.6 \%$ vs. $25.9 \%, \mathrm{p}=$ $0.09)$ or hemospermia $(90.1 \%$ vs. $86.9 \%, \mathrm{p}=0.45)$. The mean duration of hematuria and rectal bleeding was significantly greater in the aspirin group compared to the control group (4.45 \pm 2.7 vs. $2.4 \pm 2.6, \mathrm{p}=<0.001$ and $3.3 \pm 1.3$ vs. $1.9 \pm 0.7$, $\mathrm{p}<0.001$ ). Multivariate logistic regression analysis revealed that only younger patients (mean age $60.1 \pm 5.8$ years) with a lower body mass index $\left(<25 \mathrm{~kg} / \mathrm{m}^{2}\right)$ receiving aspirin were at a higher risk (odds ratio $\left.=3.46, \mathrm{p}=0.047\right)$ for developing hematuria and rectal bleeding after the procedure.

Conclusions: The continuing use of low-dose aspirin in patients undergoing extended prostatic biopsy is a relatively safe option since it does not increase the morbidity of the procedure.
\end{abstract}

Key words: prostate; biopsy; transrectal; aspirin

Int Braz J Urol. 2010; 36: 308-16

\section{INTRODUCTION}

Due to the widespread clinical use of prostate specific antigen (PSA) for the early diagnosis of prostate cancer, transrectal ultrasound-guided prostate biopsy has emerged as one of the most frequently performed urological procedures.

Reports published during the last decade have demonstrated that the classical sextant prostate biopsy scheme (as proposed by Hodge in 1989) demonstrates a false negative rate of $19-31 \%$ (1). Based on these findings, various biopsy schemes have been proposed, in order to increase the sensitivity of the method. In current clinical practice, 10-core or 12-core biopsy protocols perform better, compared to the sextant scheme, due to their increased sensitivity and relatively low complication rates (2-4).

Older patients constitute the main target group for prostate cancer screening and subsequently undergo prostatic biopsy. At the same time, cardiovascular disease most commonly affects the elderly and low-dose acetylsalicylic acid (ASA, $100 \mathrm{mg}$, 
once daily) is the mainstay of primary and secondary prophylaxis for patients with coronary and peripheral vascular disease.

The optimal management of patients who receive low doses $(100 \mathrm{mg})$ of acetylsalicylic acid (ASA) and who are scheduled to undergo prostatic biopsy is controversial. The approaches being implemented in everyday clinical practice vary and include discontinuation of ASA, replacement of ASA with low-molecular weight heparin and continuing ASA during the peri-procedural period $(5,6)$.

The aim of this study is to determine whether the incidence of hemorrhagic complications after an extended-scheme (12 or more cores) prostatic biopsy is increased for patients who continue to take low-dose ASA compared to patients not receiving ASA.

\section{MATERIALS AND METHODS}

The study was approved by the Local Research Ethics Committees. Between February 2007 and September 2008, after written informed consent was received, 530 patients were considered for prostate biopsy and were included in the protocol database. Indications for biopsy included PSA value greater than $2.5 \mathrm{ng} / \mathrm{mL}$ and/or an abnormal digital rectal examination. Exclusion criteria included: treatment with coumadin or antiplatelet drugs (clopidogrel, triflusal), a personal history of hemorrhagic diathesis, inflammatory bowel disease, malignancy in other pelvic organs, chronic liver disease, renal failure and concomitant rectal diseases such as polyps, rectal fissures, hemorrhoids and anal strictures.

\section{Biopsy Protocol}

All patients received an antibiotic prophylaxis with $150 \mathrm{mg}$ netilmicin intramuscularly before biopsy and $500 \mathrm{mg}$ ciprofloxacin twice a day administered one day before and for 4 days after the biopsy. Patients under ASA treatment were instructed to continue its use before and after the procedure.

A biplanar transrectal probe (Pro-Focus $2202^{\mathrm{TM}}$, B-K Medical, Denmark), with capability of real-time three-dimensional imaging was used. The biopsy needle was $18 \mathrm{G}$ in diameter and tissue cores were obtained by using an automated biopsy gun (Pro-Mag ${ }^{\mathrm{TM}}$ ). Intramuscular dextropropoxyphene hydrochloride administered 30 minutes prior to biopsy and local application of lidocaine gel $2 \%$ were used for peri-procedural pain control. During transrectal ultrasound scanning of the gland, the anatomic capsule, the posterolateral peripheral zone and the junction of the prostatic base with the seminal vesicles were thoroughly visualized as areas of high incidence of cancer and the presence of hypoechoic regions was noted. All the biopsies were performed by the same consultant urologist. Biopsy protocol included a standard systematic 12-core scheme (6 cores per lobe). Where deemed necessary by ultrasound findings (e.g. specific hypoechoic lesions), more additional targeted biopsies were obtained.

\section{Morbidity Assessment}

Two questionnaires were used to assess patient characteristics, clinical and laboratory features and patient- and physician-reported morbidity of the procedure. The first questionnaire was completed by the treating physician after the biopsy and included items such as prostate volume and number of biopsy cores, PSA and free PSA values, body mass index (BMI) calculation and immediate complications. The second questionnaire included questions regarding the late occurrence and duration of hemorrhagic or other complications (urinary retention, fever, etc.) and the need for a doctor's consultation or for hospitalization due to a biopsy-related problem, as well as the overall burden which was imposed on the patient's quality of life. This questionnaire was completed by the patients one month later, during a scheduled re-evaluation, according to the department's policy.

Rectal bleeding was defined as spontaneous or defecation-associated blood loss. Hematuria was graded as mild (intermittent, absence of blood clots, lasting less than 48 hours), moderate (presence of clots and involving more than $50 \%$ of voids for two to five days) and severe (acute retention due to clots, need for patient hospitalization). Pain was evaluated by using the 1-10 numeric rating scale (NRS). 


\section{Statistical Analysis}

Statistical Analysis was done with the SPSS software package (SPSS 13.0 Inc, Chicago, IL). Univariate analyses were performed, using Student's-ttest and Mann-Whitney U test for continuous variables and Chi-square test or Fisher's exact test for categorical variables. A multiple logistic regression model was designed to determine the factors (age, number of biopsy cores, PSA, prostate volume, BMI) associated with hemorrhagic complications. To find the best model, a backward elimination stepwise procedure was carried out so that the factor would be eliminated from the analysis if the corresponding $P$ value was greater than 0.15 . A two tailed $p$ value of 0.05 or less was considered statistically significant.

\section{RESULTS}

The procedure was interrupted in eight patients due to bradycardia and hypotension, and these patients were excluded from the study. A total of 434 patients fulfilled the inclusion criteria, fully completed the questionnaire and returned for scheduled re-evaluation. Of them, 152 were under ASA treatment on a daily basis and were instructed to continue ASA before and after undergoing the procedure (Aspirin Group), while 282 patients did not receive ASA (Control Group). The most common reason for taking aspirin was primary or secondary prevention of coronary heart disease $(48.6 \%)$ and the prevention of graft occlusion after coronary artery bypass grafting (11.7\%).
The mean duration of aspirin therapy was $19.6 \pm 11.7$ (range 1-43) months.

Demographic and clinical characteristics of the patients were comparable between the two groups (Table-1). The mean number of biopsy cores obtained per patient did not vary significantly between the two groups $(12.9 \pm 1.6$ versus $13.1 \pm 1.2$ cores, $p=$ $0.09)$. The histopathology examination revealed the presence of prostate cancer in 149 patients (34.3\%). The biopsy was considered well tolerated by the vast majority of patients. The mean score on the NRS was 2.1 and 2.3 for the aspirin and control group, respectively.

Regarding hemorrhagic complication rates, there were no statistically significant differences between the two groups (Table-2). There was no significant difference in the hematuria rate between patients under ASA $(64.5 \%)$ and patients not taking aspirin $(60.6 \%)(\mathrm{p}=0.46)$. Rectal bleeding rates $(33.5 \%$ versus $25.9 \%, p=0.09)$ and hemospermia rates $(90.1 \%$ versus $86.9 \%, p=0.45)$ were also comparable. Further analysis of hematuria severity, revealed the absence of statistically significant differences; $77.5 \%$ of the patients in the Aspirin group and $85.4 \%$ of the patients in the Control group reported mild hematuria $(p=0.13)$, while hematuria was graded as moderate in the remaining $22.5 \%$ and $14.6 \%$ of the Aspirin and Control groups, respectively $(\mathrm{p}=0.13)$. However, statistically significant differences were noted in terms of duration of bleeding biopsy-related events. The mean duration of hematuria and rectal bleeding was significantly higher in the Aspirin group, compared to the control group. The mean duration of hematuria was $4.45 \pm 2.7$ and $2.4 \pm 2.6$ days for the aspirin and

Table 1 -Demographic and clinical characteristics of patients.

\begin{tabular}{lccc}
\hline & $\begin{array}{c}\text { Aspirin Group } \\
(\mathbf{N}=\mathbf{1 5 2})\end{array}$ & $\begin{array}{c}\text { Control Group } \\
(\mathbf{N}=\mathbf{2 8 2})\end{array}$ & p Value \\
\hline Age & 65.4 & 64.3 & 0.12 \\
BMI $\left(\mathrm{kg} / \mathrm{m}^{2}\right)$ & 28.5 & 28.1 & 0.16 \\
Prostate volume $(\mathrm{mL})$ & 51 & 52.1 & 0.14 \\
Number of cores & 12.9 & 13.1 & 0.09 \\
PSA $(\mathrm{ng} / \mathrm{mL})$ & 7.4 & 7.3 & 0.23 \\
\hline
\end{tabular}

$B M I=$ body mass index; PSA = prostate specific antigen. 
Table 2 - Incidence and duration of hemorrhagic complications.

\begin{tabular}{lccc}
\hline Complications & Aspirin Group & Control Group & p Value \\
\hline Hematuria & $64.5 \%(98 / 152)$ & $60.6 \%(171 / 282)$ & 0.46 \\
Duration of hematuria (days) & $4.45 \pm 2.7$ & $2.4 \pm 2.6$ & $<0.001$ \\
Severity of hematuria & & & \\
$\quad$ Mild & $77.5 \%(76 / 98)$ & $85.4 \%(146 / 171)$ & 0.13 \\
$\quad$ Moderate & $22.3 \%(22 / 98)$ & $14.6 \%(25 / 171)$ & 0.13 \\
$\quad$ Severe & 0 & 0 & \\
& & & \\
Rectal bleeding & $33.5 \%(51 / 152)$ & $25.9 \%(73 / 282)$ & 0.09 \\
Duration of rectal bleeding (days) & $3.3 \pm 1.3$ & $1.9 \pm 0.7$ & $<0.001$ \\
Hemospermia* & $90.1 \%(91 / 101)$ & $86.9 \%(159 / 183)$ & 0.45 \\
Duration of hemospermia & $21.2 \pm 11.9$ & $22.4 \pm 10.4$ & 0.67 \\
\hline
\end{tabular}

*Patients who reported intercourse within the first month.

control groups, respectively ( $\mathrm{p}<0.001$ ), while the mean duration of rectal bleeding was $3.3 \pm 1.3$ and $1.9 \pm 0.7$ days, respectively $(\mathrm{p}<0.001)$. The duration of hemospermia, however, did not vary significantly between the two subsets $(21.2 \pm 11.9$ days and 22.4 \pm 10.4 days, respectively, $\mathrm{p}=0.67$ ).

Further data evaluation with multiple logistic regression analysis, revealed that a specific subgroup of younger patients under ASA (mean age $60.1 \pm 5.8$ years) with a lower BMI $\left(<25 \mathrm{~kg} / \mathrm{m}^{2}\right)$ had a higher probability to develop hematuria and/or rectal bleeding, compared to older patients (mean age $70.7 \pm$ 4.2 years) with a higher BMI $\left(>25 \mathrm{~kg} / \mathrm{m}^{2}\right)$. This difference, however was only marginally statistically significant (odds ratio $=3.46, \mathrm{p}=0.047$ ) $($ Table-3).

The evaluation of overall complication rates also revealed the absence of any significant early or late complications or biopsy-related hospitalizations.
Finally, none of the patients reported any significant burden on quality of life because of the biopsy or the post-procedural bleeding complications.

\section{COMMENTS}

Ultrasound-guided transrectal biopsy of the prostate is considered nowadays the "gold standard" for the diagnosis of prostate cancer. In everyday clinical practice, it constitutes a relatively safe and well tolerated procedure, performed on an outpatient basis. The most frequent complications observed are hematuria, rectal bleeding and hemospermia. The optimal method for peri procedural pain control has been the topic of many studies. A recent study by Tobias-Machado et al. concluded that periprostatic local anesthesia combined with low-dose sedation

Table 3 - Multivariate analysis of predictors of bleeding in patients on low-dose aspirin.

\begin{tabular}{lccc}
\hline Parameter & Exp (B) & $\begin{array}{c}\mathbf{9 5 \%} \text { CI for Exp (B) } \\
\text { Lower-Upper }\end{array}$ & p Value \\
\hline PSA & 0.878 & $0.748-1.029$ & 0.10 \\
Number of biopsy cores & 0.976 & $0.666-1.431$ & 0.90 \\
Prostate volume & 0.998 & $0.968-1.033$ & 0.89 \\
Age $<65$ years $+\mathrm{BMI}<25 \mathrm{~kg} / \mathrm{m}^{2}$ & 3.46 & $1.017-11.817$ & 0.047 \\
\hline
\end{tabular}

$\operatorname{Exp}(B)=$ odds ratio; $C I=$ confidence Interval; $B M I=$ body mass index. 
provide an effective and safe option (7). We used intramuscular dextropropoxyphene hydrochloride administered 30 minutes prior to biopsy and local application of lidocaine gel $2 \%$ with satisfactory pain management.

In this study, the rates of hemorrhagic complications were evaluated using a questionnaire that was completed by the patients one month after the biopsy during a scheduled re-evaluation. In order to diminish recall bias, before discharge, the patients were specifically instructed to be aware of any bleeding complication and to note its duration. Rates of hemorrhagic complications, as reported in prospective studies that included more than 100 patients and 6-12 prostate biopsy cores per patient, vary widely (8-11) (10-74\% for hematuria, $1-40 \%$ for rectal bleeding and $10-78 \%$ for hemospermia). These wide variations can be attributed to different methods for evaluation of complication rates, differences in complication definition, selection bias during the recruitment of patients for the study and patient preoccupation with bleeding complications because of pre-procedural informed consent. Severe and potentially life-threatening complications such as parasympatheticotonia, sepsis and uncontrollable bleeding occur at a rate of $1-2 \%$. These complications, however, can be prevented by detailed history, excellent knowledge of the ultrasonographic anatomy of the prostate and appropriate antibiotic prophylaxis.

Currently, ASA is the antiplatelet agent of choice, due to its relative safety, efficacy and low cost. For most elective surgeries, it has typically been recommended that the patient stop taking ASA 7 to 10 days before the procedure. The optimal management of patients who are scheduled for prostate biopsy and receive ASA is controversial. The lack of solid evidence contributes to the implementation of various or even controversial approaches regarding the management of these patients. Due to the estimation that the number of transrectal prostate biopsies performed is going to increase in the near future, the need for evidence-based recommendations regarding these patients is more than apparent.

On the other hand, there is evidence in recent reports that coronary patients who abruptly discontinue ASA treatment are at increased risk for a new acute vascular event (12). Acute cardiac events are noted within 10 days of ASA withdrawal and are due to coronary thrombosis. There are also clinical and experimental data that the "rebound thrombosis phenomenon" increases cerebrovascular event rates as well (13). These findings support the recommendation to restart ASA treatment within 8-10 days after a major procedure for which ASA withdrawal was deemed necessary. Burger et al. published a meta-analysis in 2005 (14) which highlighted the fact that patients under ASA treatment are exposed to a higher risk of bleeding complications during most procedures, but aspirin does not lead to a higher level of severity of bleeding complications (with the exceptions of intracranial surgery and possibly transurethral prostatectomy). These findings suggest that only patients scheduled for intracranial procedures and transurethral prostatectomy should be exposed to the increased cardiovascular risk associated with ASA withdrawal.

According to our findings, patients who undergo extended prostatic biopsy while on ASA treatment are not at increased risk for bleeding complications, compared to patients not taking ASA. This finding has been noted in previous reports, which however involved less biopsy cores per patient (6core or 8-core biopsy schemes) $(15,16)$. One other study by Halliwell et al. demonstrated an increased risk of minor but not major bleeding complications in patients taking ASA (17). The relatively low incidence of hemorrhagic events can be attributed to the phenomenon of individual patient resistance to ASA in low $(100 \mathrm{mg})$ doses, which results in treatment failure in up to $30 \%$ of patients $(18,19)$. In addition, ASA is considered an antiplatelet agent of low potency, leading to the recommendation for combining ASA with a second antiplatelet agent, such as clopidogrel, for patients at high risk for cardiovascular events (20). In our study, an interesting finding was that patients of younger age $\left(<65\right.$ years) and lower BMI $\left(<25 \mathrm{~kg} / \mathrm{m}^{2}\right)$ had a higher probability for having bleeding, as opposed to older patients ( $>70$ years) with higher BMI $\left(>25 \mathrm{~kg} / \mathrm{m}^{2}\right)$. This difference, however, was statistically marginal. Several reports suggested that older and obese patients are more prone to the development of aspirin resistance $(21,22)$. One can suggest that ASA antiplatelet effect is more pronounced in younger patients with lower BMI or that the optimal dose of ASA is BMI-dependent. 


\section{CONCLUSION}

Continued low-dose ASA treatment is not associated with an increased incidence of hemorrhagic complications after an extended (12 or more cores) prostatic biopsy and therefore ASA withdrawal is not considered justified. Prospective randomized trials are needed, in order to provide solid data for evidencebased recommendations.

\section{CONFLICT OF INTEREST}

None declared.

\section{REFERENCES}

1. Terris MK: Sensitivity and specificity of sextant biopsies in the detection of prostate cancer: preliminary report. Urology. 1999; 54: 486-9.

2. Berger AP, Gozzi C, Steiner H, Frauscher F, Varkarakis J, Rogatsch H, et al.: Complication rate of transrectal ultrasound guided prostate biopsy: a comparison among 3 protocols with 6,10 and 15 cores. J Urol. 2004; 171: 1478-80; discussion 1480-1.

3. Mariappan P, Chong WL, Sundram M, Mohamed SR: Increasing prostate biopsy cores based on volume vs the sextant biopsy: a prospective randomized controlled clinical study on cancer detection rates and morbidity. BJU Int. 2004; 94: 307-10.

4. Nesrallah L, Nesrallah A, Antunes AA, Leite KR, Srougi M: The role of extended prostate biopsy on prostate cancer detection rate: a study performed on the bench. Int Braz J Urol. 2008; 34: 563-70; discussion 570-1.

5. Masood J, Hafeez A, Calleary J, Barua JM: Aspirin use and transrectal ultrasonography-guided prostate biopsy: a national survey. BJU Int. 2007; 99: 965-6.

6. Connor SE, Wingate JP: Management of patients treated with aspirin or warfarin and evaluation of haemostasis prior to prostatic biopsy: a survey of current practice amongst radiologists and urologists. Clin Radiol. 1999; 54: 598-603.

7. Tobias-Machado M, Verotti MJ, Aragao AJ, Rodrigues AO, Borrelli M, Wroclawski ER: Prospective randomized controlled trial comparing three different ways of anesthesia in transrectal ultrasound-guided prostate biopsy. Int Braz J Urol. 2006; 32: 172-9; discussion 179-80.
8. Raaijmakers R, Kirkels WJ, Roobol MJ, Wildhagen MF, Schrder FH: Complication rates and risk factors of 5802 transrectal ultrasound-guided sextant biopsies of the prostate within a population-based screening program. Urology. 2002; 60: 826-30.

9. Peyromaure M, Ravery V, Messas A, Toublanc M, Boccon-Gibod L, Boccon-Gibod L: Pain and morbidity of an extensive prostate 10-biopsy protocol: a prospective study in 289 patients. J Urol. 2002; 167: 218-21.

10. Mkinen T, Auvinen A, Hakama M, Stenman UH, Tammela TL: Acceptability and complications of prostate biopsy in population-based PSA screening versus routine clinical practice: a prospective, controlled study. Urology. 2002; 60: 846-50.

11. Djavan B, Waldert M, Zlotta A, Dobronski P, Seitz C, Remzi M, et al.: Safety and morbidity of first and repeat transrectal ultrasound guided prostate needle biopsies: results of a prospective European prostate cancer detection study. J Urol. 2001; 166: 856-60.

12. Ferrari E, Benhamou M, Cerboni P, Marcel B: Coronary syndromes following aspirin withdrawal: a special risk for late stent thrombosis. J Am Coll Cardiol. 2005; 45: 456-9.

13. Maulaz AB, Bezerra DC, Michel P, Bogousslavsky J: Effect of discontinuing aspirin therapy on the risk of brain ischemic roke. Arch Neurol. 2005; 62: 1217-20.

14. Burger W, Chemnitius JM, Kneissl GD, Rücker G: Low-dose aspirin for secondary cardiovascular prevention - cardiovascular risks after its perioperative withdrawal versus bleeding risks with its continuation - review and meta-analysis. J Intern Med. 2005; 257 . 399-414.

15. Maan Z, Cutting CW, Patel U, Kerry S, Pietrzak P, Perry MJ, et al.: Morbidity of transrectal ultrasonography-guided prostate biopsies in patients after the continued use of low-dose aspirin. BJU Int. 2003; 91 : 798-800.

16. Giannarini G, Mogorovich A, Valent F, Morelli G, De Maria M, Manassero F, et al.: Continuing or discontinuing low-dose aspirin before transrectal prostate biopsy: results of a prospective randomized trial. Urology. 2007; 70: 501-5.

17. Halliwell OT, Yadegafar G, Lane C, Dewbury KC: Transrectal ultrasound-guided biopsy of the prostate: aspirin increases the incidence of minor bleeding complications. Clin Radiol. 2008; 63: 557-61.

18. Hankey GJ, Eikelboom JW: Aspirin resistance. Lancet. 2006; 367: 606-17.

19. Michos ED, Ardehali R, Blumenthal RS, Lange RA, Ardehali H: Aspirin and clopidogrel resistance. Mayo Clin Proc. 2006; 81: 518-26. 
20. Yusuf S, Zhao F, Mehta SR, Chrolavicius S, Tognoni G, Fox KK, et al.: Effects of clopidogrel in addition to aspirin in patients with acute coronary syndromes without ST-segment elevation. N Engl J Med. 2001; 345: 494-502. Erratum in: N Engl J Med 2001; 345: 1716. N Engl J Med 2001; 345: 1506.

21. Greenblatt DJ, Abernethy DR, Boxenbaum HG, Matlis R, Ochs HR, Harmatz JS, et al.: Influence of age, gen- der, and obesity on salicylate kinetics following single doses of aspirin. Arthritis Rheum. 1986; 29: 971-80.

22. Tamminen M, Lassila R, Westerbacka J, Vehkavaara $\mathrm{S}$, Yki-Järvinen H: Obesity is associated with impaired platelet-inhibitory effect of acetylsalicylic acid in nondiabetic subjects. Int J Obes Relat Metab Disord. 2003; 27: 907-11.
Accepted after revision:

November 03, 2009

\section{Correspondence address:}

Dr. Demetrios Delakas

Department of Urology

Asklipieion General Hospital

1, Vasileos Pavlou Avenue

16673, Voula, Athens, Greece

Fax: + $30210892-3111$

E-mail: delakas@mail.gr

\section{EDITORIAL COMMENT}

The present study suggest that the continued use of low-dose aspirin (LDA) in patients undergoing TRUS-guided prostate biopsy does not increase the incidence of mild bleeding complications, it only prolongs the duration of self-limited hematuria and rectal bleeding.

This study was not devoid of limitations. First, most patients take LDA as a prophylactic agent for coronary and peripheral vascular diseases and a control group of patients with discontinuing LDA was necessary to compare patients with the same comorbities. Second, the question of whether the longer duration of hematuria and rectal bleeding in men who continued LDA is clinically significant might have been addressed by a comparison of the hemoglobin levels before and after prostate biopsy. Third, the duration of hematospermia, which can persist for up to 2 months after prostate biopsy, was not recorded after 30 days (1).

There are no guidelines on the management of LDA before taking prostate biopsies but there appears to be no strong scientific evidence for the withdrawal of aspirin in all patients undergoing prostate biopsy.

In one study, $52 \%$ of radiologists and $27 \%$ of urologists terminated aspirin before prostate biopsy (2). In the UK, $35 \%$ of urologists routinely stop aspirin before prostate biopsy (3).

\section{REFERENCES}

1. Lee G, Attar K, Laniado M, Karim O: Safety and detailed patterns of morbidity of transrectal ultrasound 
guided needle biopsy of prostate in a urologist-led unit. Int Urol Nephrol. 2006; 38: 281-5.

2. Connor SE, Wingate JP: Management of patients treated with aspirin or warfarin and evaluation of haemostasis prior to prostatic biopsy: a survey of cur- rent practice amongst radiologists and urologists. Clin Radiol. 1999; 54: 598-603.

3. Masood J, Hafeez A, Calleary J, Barua JM: Aspirin use and transrectal ultrasonography-guided prostate biopsy: a national survey. BJU Int. 2007; 99: 965-6.

Dr. Valdemar Ortiz

Chair, Section of Urology

Federal University of Sao Paulo, UNIFESP

Sao Paulo, SP, Brazil

E-mail:valdor@uol.com.br

\section{EDITORIAL COMMENT}

Although prostate biopsy is widespread and regarded as a routine office procedure, some men are probably harmed. Kariotis et al. (1) evaluated whether the continuing use of low-dose aspirin in patients undergoing extended prostate biopsy is a safe option. The authors should be congratulated for the clarity of the text, the excellent analysis, and the cautious interpretation of their results.

The goals of prostate biopsy are to avoid biopsy-associated complications such as bleeding, infection and pain as well as to detect prostate cancer effectively. Especially, patient safety is a worldwide concern. Every medical and surgical interventions have benefits and risks. Patients and physicians deserve to be fully informed about the risks as well as benefits.

The use of aspirin was already found to be a relatively safe option in patients undergoing extended prostate biopsy (2-6). However, some investigators reported that aspirin prolonged the duration of hematuria and rectal bleeding (7) or exacerbated minor bleeding complications (8). Therefore, to confirm whether patients taking aspirin were more likely to experience bleeding complications in routine clinical setting, as admitted by the authors, prospective randomized studies should be designed.

\section{REFERENCES}

1. Kariotis I, Philippou P, Volanis D, Serafetinides E, Delakas D: Safety of ultrasound-guided transrectal extended prostate biopsy in patients receiving low-dose aspirin. Int Braz J Urol (in press).

2. Rodríguez LV, Terris MK: Risks and complications of transrectal ultrasound guided prostate needle biopsy: a prospective study and review of the literature. J Urol. 1998; 160: 2115-20.

3. Herget EJ, Saliken JC, Donnelly BJ, Gray RR, Wiseman D, Brunet G: Transrectal ultrasoundguided biopsy of the prostate: relation between ASA use and bleeding complications. Can Assoc Radiol J. 1999; 50: 173-6.

4. Maan Z, Cutting CW, Patel U, Kerry S, Pietrzak P, Perry MJ, et al.: Morbidity of transrectal ultrasonography-guided prostate biopsies in patients after the continued use of low-dose aspirin. BJU Int. 2003; 91: 798-800.

5. de Jesus CM, Corrêa LA, Padovani CR: Complications and risk factors in transrectal ultrasoundguided prostate biopsies. Sao Paulo Med J. 2006; 124: 198-202.

6. Ecke TH, Gunia S, Bartel P, Hallmann S, Koch $\mathrm{S}$, Ruttloff J: Complications and risk factors of transrectal ultrasound guided needle biopsies of 
the prostate evaluated by questionnaire. Urol Oncol. 2008; 26: 474-8.

7. Giannarini G, Mogorovich A, Valent F, Morelli G, De Maria M, Manassero F, et al.: Continuing or discontinuing low-dose aspirin before transrectal prostate biopsy: results of a prospective randomized trial. Urology. 2007; 70: 501-5.
8. Halliwell OT, Yadegafar G, Lane C, Dewbury KC: Transrectal ultrasound-guided biopsy of the prostate: aspirin increases the incidence of minor bleeding complications. Clin Radiol. 2008; 63: 557-61.

Dr. Jae-Seung Paick

Department of Urology

Seoul National University Hospital

Seoul, Korea

E-mail: jspaick@snu.ac.kr 